\title{
Regulation and Standardization of Digital Technologies Used in Facilitating the Mitigation of COVID-19 Contagion
}

\author{
James Kunle Olorundare ${ }^{1, *}$, Adebimpe Olubunmi Olorundare ${ }^{2}$ \\ ${ }^{1}$ Department of Technical Standards and Network Integrity, Nigerian Communications Commission, Abuja, Nigeria \\ ${ }^{2}$ Department of Information Management System, Netquist Nigeria Limited, Abuja, Nigeria
}

Email address:

olorundarek@ncc.gov.ng (J. K. Olorundare)

${ }^{*}$ Corresponding author

\section{To cite this article:}

James Kunle Olorundare, Adebimpe Olubunmi Olorundare. Regulation and Standardization of Digital Technologies Used in Facilitating the Mitigation of COVID-19 Contagion. International Journal of Sustainable Development Research. Vol. 7, No. 1, 2021, pp. 1-8. doi: $10.11648 /$ j.ijsdr.20210701.11

Received: December 31, 2020; Accepted: January 11, 2021; Published: January 30, 2021

\begin{abstract}
The paper discusses practical applications of digital technologies (e.g. Artificial Intelligence, Internet of Things, Big Data Analytics, Mobile Technologies etc.) on how COVID-19 pandemic and other contagion curve can be flattened. The background of the paper deals with issues around the novel COVID-19, the way different countries responded to it generally but with a focus on the application of digital technologies and its effects. The objective of the paper is to show how important digital technologies are being used to help flatten the curve of COVID-19 pandemic. More so, analysis of the digital technologies was also carried out. The methodology was hinged on facts analysis and observations based on what needs to be improved while leveraging on digital infrastructures to flatten the COVID-19 infection rate curve. Under the methodology, studies were conducted on randomly sampled countries which were analyzed and specific digital technologies were identified for regulations and standardization. Recommendations were made based on studies conducted in countries like South Korea, Taiwan etc. In conclusion, ICT related policies were identified and specific innovative digital technologies were identified for regulatory interventions and future standardization works. The candidate digital technologies identified are proposed for technical studies by relevant standards bodies e.g. International Telecommunications Union-Standardizations (ITU-T) is expected to create relevant Study Question based on the identified digital technologies and carry out studies leading to the standardization of the digital technologies. This will accelerate the flattening future contagion infection rate curve.
\end{abstract}

Keywords: Mobile Technologies, Industry 4.0, Big Data Analytics, Artificial Intelligence (AI), Internet of Things (IoT), COVID-19, E-readiness, E-health/Ehealth, ITU-T

\section{Introduction}

Electronic readiness is the level of preparedness and the ability of a particular country to be involved in the electronic world businesses. It can also refer to a country's capacity and state of preparedness to participate in the electronic world. The maturity status can be measured by the infrastructure put in place by a particular country in terms of Information and Communications Technology (ICT) infrastructures and the capacity or the ability of the country to optimally utilize the ICT infrastructures to impact the economy and the social life of the nationality positively $[1,2]$. This may include using it to facilitate health sector of the economy and the ability to deploy the ICT capacity during emergency which may include a contagion like COVID-19.

The ability of a country to use the infrastructure positively can be easily tested during a pandemic like the COVID-19. This performance of the country can serve as a measure to evaluate the electronic preparedness and this may vary from country to country or even depending on the level of analysis and considering the extant standards for disaster management or business continuity management as the case may be. In the two considerations, it can be observed that the classes of infrastructure in such an environment would determine how fast the pandemic can be easily mitigated because of the benefits that can be derived from the applications and the use of ICT facilities depending on availability, standards, 
regulatory considerations etc.

The paper will do in-depth analysis and discussion on the variable options and point out the necessity of future standardization based on scenario analyses from different countries experiences. From here the paper is arranged as follows; Theoretical framework in section 2 to Literature review in 3. This is followed by methodology which is the scenario studied with analyzed countries like South Korea and Taiwan. This is followed by Recommendations in Section 5 and Future Research in Section 6 just before the references. The scope of this paper is global since the issue studied is a global pandemic and it is relevant to all countries that are members of ITU and beyond.

In conclusion, recommendations of New Work Items as proposal to ITU relevant sectors for commencement of new Work Items in the identified areas of studies were made. This include new benchmarking for standardization on Measurement of E-readiness, minimum requirements for Protocols and Standards for ICT Infrastructures used during pandemic like COVID-19, Standardizations and regulation of E-Commerce, Emergency Number so as to make the world prepared better for pandemic using the digital technologies.

\section{Theoretical Framework}

The use of digital medium to create awareness and educate the old and young in the served, unserved and underserved areas to know the right thing to do about COVID-19 pandemic is important in all sections of the society including the urban areas and suburbs. The reason is that there are people who do not believe that there is COVID-19 pandemic and some know that it exists but they do not have access to the right information as they are being fed from different sources on social media in which the facts are mixed with myth and false information. The concern of widespread of wrong information was a big issue in the early days of the pandemic. However, one thing is clear. There is no regulation or standardization on how social media can be used during pandemic without distorting the privacy of individuals. Social media has become a great influencer of the information flow in our society in the current days hence it cannot be ignored.

Mobile Technology is one of the most popular out of the trendy digital technology that has brought so much digital transformation. It is in the well served, underserved and the unserved regions. If it harnessed or regulated to channel appropriate information during pandemic, it may be a useful tool to pass broadcast or disseminate information to the three identified regions because of its social spread across our society. Mobile Technology also serve as a pedestal on top of which other applications are being harnessed especially in this 4th industrial revolution with the LTE, 4G and beyond. Mobile technology is standardized and regulated, however, the applications that run on it like apps that can be used for different applications may need to be looked at for regulations and standardization. A lot of live video applications are coming up today for different purposes which can help in maintaining social distancing, however, issues of cyber security and privacy are of concern and needs to be looked at for the purpose of regulation and standardization.

Another critical concern is how much bandwidth and other IP parameters in terms of protocols may be required before some applications can work perfectly without latency, jitter and delay bringing down the minimum Quality of Experience to a non-acceptable level.

E-Commerce based on the availability of digital infrastructure helps in reducing crowding in centers such as supermarkets and malls. It can facilitate and assist in maintaining social distancing for the purpose of keeping the COVID-19 pandemic curve flat. However, many people do not really follow the social distancing rule which of course made the situation to aggravate. The use of Automated Teller Machine during pandemic period may be a medium of spreading the COVID-19. The use of online transaction for payment can help reduce the withdrawal of cash thereby help in reducing COVID-19 infection rate.

Social Media and Apps: there are many social media apps in circulation. They are being used to spread information but it is believed that these can be harnessed as a digital medium to mitigate the effect of pandemic such as COVID-19. These can be used on both mobile terminal and fixed devices like desktop, smart television etc.

Despite all these technologies highlighted above which have brought digital transformation, there are challenges in optimizing them especially during a pandemic situation due to regulatory issues, standardization and policies which need to be put in place to harness them in fighting pandemic such as COVID-19. Pandemic, such as COVID-19 needs to be prepared for based on availability of digital infrastructures and technologies. Based on the foregoing, this paper is written with the objectives to identify and propose regulatory interventions such as drafting relevant policies, standards and regulation in line with digital transformation for the purpose of using such document during a pandemic. The key research question is: What are those digital transformation technologies that need to be regulated and or standardized for the purpose of combatting COVID-19? The intent of the research is to contribute to the body of knowledge by proposing New Question and New Work Items for studies by relevant ITU Study Groups with respect to combatting pandemic such as COVID-19 using Industry 4.0 tools. This paper was written during the COVID-19 pandemic.

\section{Literature Review}

Degree of preparedness of a country's government, citizens, businesses and non-governmental entities to use ICT for sustainable development through the use of communication technologies and telecommunication infrastructures can be said to be e-readiness. It goes to include the migration of traditional businesses to be able to use ICT infrastructures unhindered $[1,2]$. In other words it can be seen as the positioning of a society to take advantage of opportunities 
that ICT infrastructures can offer to run economic activities, businesses and other essential services which can be run without the use of ICT but because of the available infrastructures such businesses are more effective when they are run using the ICT infrastructures

This can include the readiness to combat pandemic like COVID-19. COVID-19 pandemic has spread to all the continents of the world and many countries and this is stretching the health system of all the countries where the infection has spread to. It is an understatement that many countries health workers cannot cope with the number of infection and there is need to find alternative way to combat the pandemic through the use of ICT infrastructures and applications related to the Industry 4.0. The IP networks based on the on TCP/IP protocols are part of the fundamentals of Internet which is an integral part of the 4th Industrial Revolution and it contributes immensely as an essential infrastructure when matrices of e-readiness should be specified because without the Internet, e-readiness may not be complete based on the authors' assertions.

The E-Health is seen as capacity improvement and advancement of the extant traditional health system through the utilization of ICT infrastructure to improve the traditional health system. The focus of E-health in any care provision in terms of the type of services provision and the reach of served, the underserved and possibly the unserved citizenry so as to have access to health care according to Sustainable Development Goal 3 which is: "Ensure healthy lives and promote wellbeing for all at all ages" [3], This can include connecting intra-state hospital, inter-state hospitals, interregional hospital and even inter-continental hospitals especially from an advanced hospitals with advanced expertise and specialization in a particular field to a location without such an expertise.

E-health in this paper can be used as e-health which is also electronic health. The major objective of E-health is to promote and strengthen the use of ICT in health development from field applications to hospital procedure applications with network connectivity across two or more different locations for optimizing usage of advance specializations. E- Health is a new healthcare strategy which is still evolving all over the world. However, COVID-19 has created a disruptive milestone pushing many countries to accelerate the deployment of ehealth. There are challenges in the deployment because ehealth cannot be executed without ICT infrastructure but the ereadiness of many countries if measured based on availability of infrastructures may still be low. This type of measurement needs to be standardized so that the definition would be the same if recommended by for instance, ITU-T.

There is appreciable development in health Information Technology especially with respect to sharing of information due to the seamless presence of the Internet which makes it easy and also broadband technology has brought the use of telemedicine which makes it possible for experts to be able to attend to patient in another location. This is a borderless service based on the use of Information and Communications Technologies [3]. Telemedicine would be an excellent tool to be harnessed against CORVID-19 pandemic which is already taking its toll on the medical practitioners because of many infected people to attend to in the hospital.

There is a digital divide between the developed and the developing countries of the world. This can be seen in the distribution of benefits from ICT that created global imbalance. For instance, United States of America and China account for $75 \%$ of all patents related to Blockchain technologies, $50 \%$ of global spending on IoT, $75 \%$ of the cloud computing market. In addition to that, about $90 \%$ of the market capitalization value of the world's 70 largest digital platforms. This implies that there is still huge digital divides in the world and half of the world population is still offline. This can be seen in the availability of communications infrastructures like broadband facilities and this digital divide also translate into the availability of telemedicine facilities and other technologies that can be used in the treatment of COVID-19 [4].

In a pandemic situation like COVID-19, eHealth in general, and telemedicine in particular can be a vital resource to remote regions of emerging and developing countries but it is often challenging to establish because of inadequate ICT infrastructure [5]. For instance, in this COVID-19 pandemic Nigeria is trying to set up enough laboratory to test samples and availability of enough reagent is also a challenge in Nigeria in the early days of the pandemic. The difficult financial situation in many African states implies that the majority of the African countries are poorly treated medically. In many developing countries, it is not only about insufficient facilities and trained man power, but lack of access to ehealth because of inadequate broadband facilities and access to the Internet access in unserved rural areas [5]. This is a serious challenge in time of COVID pandemic. Developed countries are also grappling with coping with the pandemic because of rate of infection and it is only feasible to rely on optimizing communications and telecommunications infrastructure to flatten the effect of COVID-19. This is what this paper intends to establish and proffer harmonized strategic solutions in terms of standardization, benchmarking of matrices, specifications of identified parameters and requirements.

Self-monitoring is the use of sensors or tools which are readily available to the general public to track and record personal data. The sensors are usually wearable devices and the tools are digitally available through mobile device applications. From a survey conducted and reported by Olorundare et all, majority of Nigerians would really want self-monitoring system which is a very good development in time of pandemic like the COVID-19. This implies that if health application is deployed using IoT monitoring systems, it will be embraced and this will help to reduce the rate of infection and in a short time, the rate of infection will be flatten through self-distancing and stay at home while those under quarantine can still be monitored without further exposing the health workers monitoring them. This is one of the advantages of self-monitoring system based on e-health [6].

Telecoms operators and other ICT service providers are 
recognizing that their customers will rely on ICT services during stay-at-home in order to flatten the rate of spread; self-isolation for those that have contacted COVID-19 and unconfirmed cases still being tested and quarantine for those with confirmed COVID-19 cases. The overarching objectives are for working at home, personal or family entertainment and maintaining social connections with others. Also, for accurate information broadcast to all.

Operators in countries like France, Australia, Saudi Arabia, Bahrain, Russia and Belgium for the purpose of illustration have started working on an increase or removal of data caps, or even free unlimited Internet especially for fixed subscribers [7]. The importance of this is for their citizens that are subscribers to those operators services to be seamlessly connected so that they can communicate in time of emergency for medical crisis or for them to be able to do away with boredom in terms of entertainments or to be able to do electronic transaction for the purpose of receiving or transferring money or to buy essentials online like food, groceries etc.

The use of computers, Personal Digital Assistant, mobile phones etc have shown that they can help to facilitate healthcare system. The positive impact can be appreciated more during pandemic situation like the COVID-19 in which social distancing is being advised and the healthcare workers are also very careful in making contacts with patients hence if there are personal Digital Assistants, mobiles phones that can be used to take vitals contactless, it will go a long way to reduce the risk of exposure thereby minimizing the infection of health workers who only need to get the readings of the vitals from patient [8].

\section{Methodology}

\subsection{Scenario Studies}

The methodology used in this research is the study of different countries scenario and qualitative analyses of the scenario to throw up what regulations need to be studied, what parameters, matrices needs to be standardized and perhaps propose as New Work Item or Questions for study by relevant ITU Sector. These were analyzed based on the understanding of the scenario to derive identified gaps and make recommendations based on the identified gaps. The countries analyzed are South Korea and Taiwan as part of this study, making up the Primary Research analysis where recommendations are brought forward for consideration based on the initial Theoretical Framework formed at the inception of the paper. Secondary research of existing literatures was also carried out to bring up the gaps which are later aligned in the scenario studies based on which valuable recommendations are drawn.

\subsection{Taiwan Scenario}

Taiwan is one of the countries that the COVID-19 pandemic affected. Taiwan is an Island just like Australia both has similar population. It was expected that Taiwan would have second highest cases of COVID-19 due to its proximity to China while Australia is farther compared to Taiwan. But the number of COVID-19 infection between the two countries differ. Taiwan: 355 to Australia 5,550 as at 4th April, 2020 (Kindly see Table 1 below). This is due to different strategies and approaches used to contain COVID19. Taiwan is very close to China with a distance of 81 miles off the coast of Mainland China. Clearly, Taiwan is more at risk due to closeness but Taiwan leant lesson from the SAR in which the infection rate was very high. More than 150,000 people were quarantined on the island during the SAR epidemic and 181 people died [9]. The lesson includes: Quick response which include close of border. In addition to that, Taiwan Policy include Policies that allow the use of Technology to track and trace COVID-19 infection. This is the success story of Taiwan.

Table 1. Comparing Taiwan and Australia Case of COVID-19.

\begin{tabular}{llllllll}
\hline S/ N & Countr $\mathbf{y}$ & Tota I Case $\mathbf{s}$ & New Case $\mathbf{s}$ & Tota I Deat h & New Deat h & Total Recover ed & Activ e case \\
\hline 1 & Taiwan & 355 & +7 & 5 & 300 & 50 \\
2 & Austral ia & 5,550 & +96 & 30 & +2 & 585 \\
\hline
\end{tabular}

Source: https://www.worldometers.info/coronavirus/ [4th April, 2020]

Taiwan citizens' household registration system allows tracking of individuals with high risk and this is also used for the foreigners coming into Taiwan based on recent travel history in affected areas. Those identified as high risk are monitored electronically through mobile phones. This is made possible due to the integration of National Health Insurance Administration and National Immigration Agency data for identification and application of big data analytics.

At the border a mobile health declaration pass can be sent using phone SMS on local telecommunications operators. Entry Quarantine System was also launched and this works by scanning a QR code that activates an online form for both departing and arriving passengers at Taiwan Airport. This accelerates immigration clearance for minimal risk travelers.
The toll-free number 1922 is a hotline deployed for reporting suspected COVID-19 cases. All these help in bringing down rate of infection in Taiwan as seen in the table below [10].

\subsection{South Korea Scenario}

South Korea used ICT tools to combat the COVID-19 pandemic as follows:

Fast-developed testing kit: Combatting COVID-19 goes beyond the use of traditional medicine. Innovations and other initiatives beyond social distancing, need to be employed to rapidly flatten the curve. To flatten the curve, South Korea has used Artificial Intelligence to develop test kits within a very short time of 3 weeks and South Korea has a testing 
capacity of 15,000 per day on average. This has helped to flatten and bring down the curve rate of infection [11].

Smart quarantine information system: This is a system that allows the monitoring of incoming passengers from epidemic countries and this information is shared with relevant agencies (Ministry of Foreign Affairs, Health Insurance
Review \& Assessment Service, telecommunication companies and the Korea Centers for Disease Control and Prevention - KCDC) in Korea as shown in Figure 1 below. The passengers are monitored within the incubation period of the disease the system is an ICT platform which uses roaming data of oversea arrival [12].

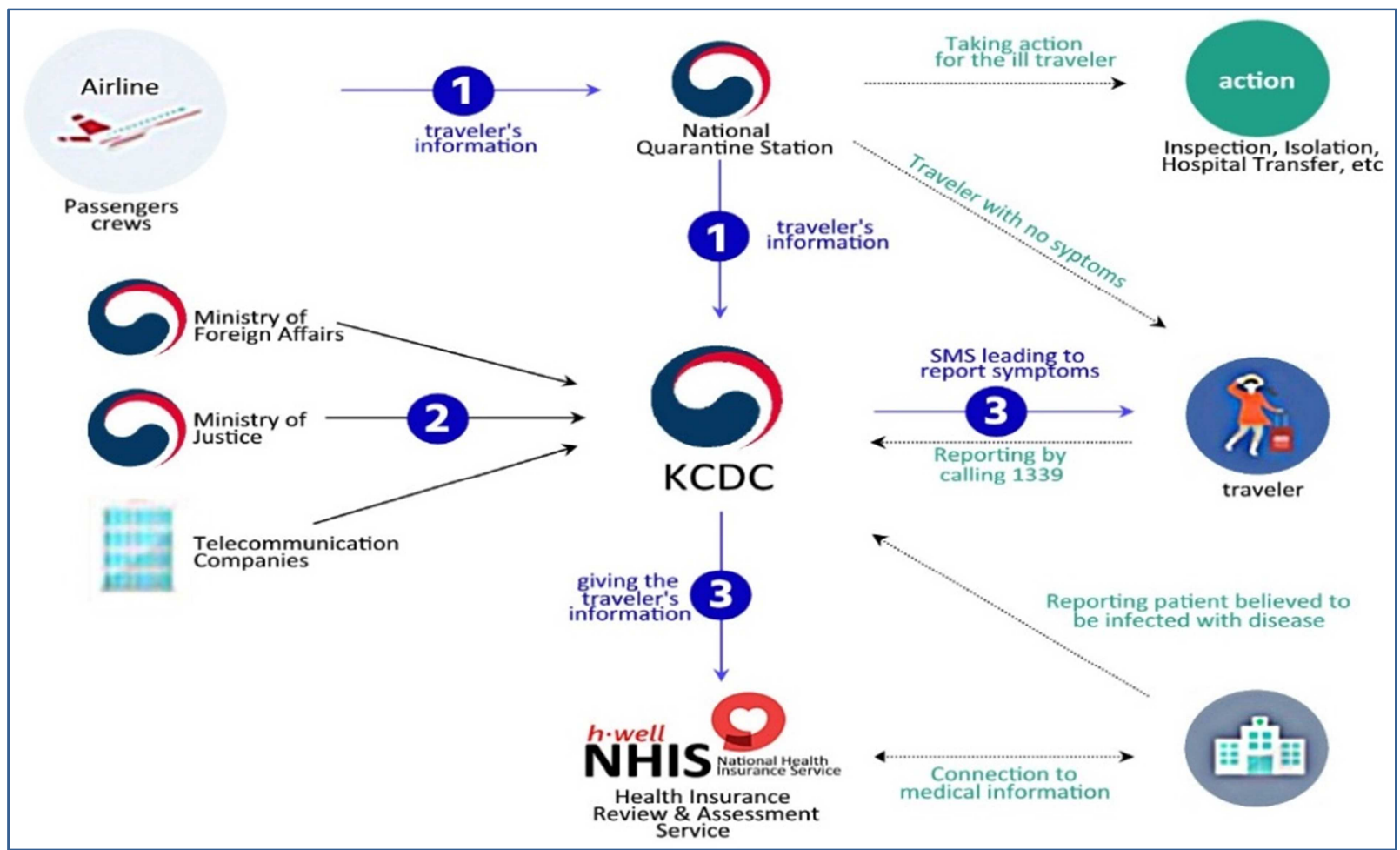

Figure 1. Smart Quarantine System.

Source: http://www.cdc.go.kr/contents.es?mid=a30301180000

[Accessed 7th April, 2020]

Mobile phone technology data for contact tracing and Mobile App for Information Sharing: Several mobile apps have been developed in a very short time which can show where to buy mask, another one can direct a patient to the closest testing center [11]. There are other one that give public critical information and so many applications. These help to combat COVID-19 and to slow down the rate of infection which was initially rapid.

AI for improving diagnosis efficiency and patient classification: The South Korea has developed a Chest XRay AI Image Support Decision Tool. This helps to categorize the cases of COVID-19 into Mild, moderate, severe and very severe, this is a risk mitigation strategy which helps in treating each case based on the category. The system is based on an algorithm for identifying abnormal findings on chest $\mathrm{x}$-rays. The systems can examine the lungs within just three seconds [11].

Daegu: Making use of a smart city hub: Daegu was the epicenter of COVID-19 in South Korea but it is now being transformed into a Smart City and the Smart Hub in the city has been used during the epidemiological investigation to create route tracing application being used to develop new medicine [11].
The country has used Artificial Intelligence and ICT infrastructures to flatten the COVID-19 Infection propagation rate curve. There are apps that can be downloaded to show where you can easily get tested, buy face mask etc. Also, The Artificial Intelligence system has been developed to make test faster. The quarantine patient are also being monitored using mobile phones applications to know their status. There is also an AI that helps to trace the route of an infected person for tracking and also for other people around the area to verify if they have come in contact with such an infected person. All this innovation using the ICT were developed based on the lesson leant from past epidemic and the smart systems, robotics and artificial intelligence are being deployed in addition to the quick response on the traditional health system. This is a similar strategy to that of Taiwan and it has yielded results in terms of flatten the COVID-19 infection rate curve [13].

\subsection{Scenario Analysis}

One thing that is common to all the countries reviewed is the fact that the infection propagation rate is increasing more which may be established based on the fact that there are 
many asymptomatic infected people that are not yet tested and are not suspected and they are still infecting people. However for countries like South Korea, and Taiwan that added full deployment of ICT Infrastructures based on past lesson learnt which made them to formulate policies on epidemic Management System the result can be seen to be flatten of the COVID-19 infection curve. Hence the need to apply other measures beyond the social distancing, stay at home, quarantines and isolation have been proved to be very effective.

From the country scenarios analyzed vis-a-vis the use of Artificial Intelligence, IC T infrastructures, Smart System have shown that to suppress the rate of spread of COVID-19, effective policies on the deployment of ICT Infrastructures,

Smart Systems, Robotics and artificial Intelligence need to be enabled. And challenges that was observed in the scenario studies was that each country was coming up with her initiative without a harmonized standards. Although, Taiwan experience was built out of the SAR experience some years back, there is still need to bring up Questions or New Work Items at the level of ITU for the purpose of Standardizations and making Recommendations which will be adopted either by Traditional Approval Process (TAP) or Alternative Approval Process (AAP) for the purpose of harmonization and general acceptability. Based on the foregoing, the following are hereby proposed for standardizations, issuance of Guideline, Recommendations by ITU-T. This can be one robust Recommendation integrating all the identified specifics together as Pandemic Management System using ICT or different Recommendations to cover for each of the specifics as it relates to their application in epidemic or pandemic Management System

\section{Recommendations}

Based on the analysis and the scenario analysis, it is recommended that there is need for ITU-T or ITU-D to create a Question that will address the issue of establishing parameters and matrices to standardize the benchmarking of e-readiness so as to make it easier to define E-readiness for all intents and purposes including measuring e-readiness for the purpose of measuring the level of e-readiness of a particular country.

It is therefore recommended that licensing of e- commerce services in order to have standardized services which are based on specific terms and conditions for the purpose of regulatory interventions during contagion or pandemic situation is hereby proposed. It is true that e- commerce is proliferating even without the emergency situation, however there is need for ITU-T to look at a proposed Work Item that will deal with standardization issues that need to be benchmarked for the purpose of e- commerce services during pandemic because of the stringent needs to adhere to some health condition. E.g. the use of robots for the preparation of the required articles so as to limit human influence in touching the packages thereby minimizing the risk of transmission of pathogen.
Other issues of concerns are the need to create specific license that will be able to carry out e-commerce during contagion in addition to the general e-commerce services for regulatory obligation such a company can be sanctioned for violations of License Conditions such as Consumer Code of Practice, concerns around Quality Assurance and set regulatory standards in terms of service rendered online. Issues such an online payment, privacy and security as it relates to the customers should be spelt out with no ambiguity. Confirmation of products quality online without manipulating the pictures and videos should be itemized, home delivery condition should be included etc. This would help in maintaining Social Distance and Stay-at-Home order during contagion like COVID-19.

If people are asked to stay at home in order to suppress the spread of COVID-19, they need to get food, the essential and other things. The application of online interactivity platform where people can do commercial activities is very critical in order to minimize human to human contact. Hence the need to have minimum acceptable standards in terms of how the services will be executed including the use of contactless Drones / Unmanned Aerial Vehicles for delivery of articles.

The need to define the minimum broadband standards and specifications needed to carry out e-commerce activities in order to prevent delay in service delivery needs to be defined too. This is due to the multimedia nature and the cybersecurity concerns i.e. E-commerce site can contain, test, photos, videos, voice note and payment medium which should be secured in terms of security. It is expected that the payment medium should not have any downtime for immediate reconciliation of payments made. These are features that need minimum requirements for effective performance.

Emergency communications has become part of standard communication system. Emergency response systems are used by organizations to assist in responding to an emergency situation that needs to be dealt with immediately. Countries also integrate national emergency network into the national communications system. These systems support communications, data gathering, data analysis, and decisionmaking of emergency response. They are rarely used but when needed, must function well and the failure rate is expected to be very low [14]. It is therefore proposed that New Work Item under relevant ITU-T Question should be created to address the minimum requirements for close to $100 \%$ success rate efficiency of Emergency Communication System.

It is recommended that a harmonized and standardized National Emergency number should be set up in each country jurisdiction so as to serve as a means of communication in emergency situation as it were in the COVID-19 pandemic so that citizens of a particular country can call for help and it should also be available to give out correct and accurate information in order to debunked wrong information being circulated through social media channels. A lot of wrong information are being pushed out by individuals during the COVID-19 pandemic through the social media platforms 
mostly under anonymity. Correct information dissemination though right central source riding on ICT platform would assist in regulating information dissemination and broadcasting. This can include the use of short code use for information dissemination for broadcast, use of QR codes for identification of individuals and access to relevant information.

Enhanced Social Media Contagion Communications: Social media has come to stay in our society and all over the world people use social media to get up to date information. Although there is need to maintain freedom of speech. however, there is need to have channels of communicating accurate information established on social media for all countries to ensure giving out standardized information so as to prevent misinformation. Countries are advised to create reliable social media links for information dissemination and to neutralize the effect of wrong information on the social media. It is proposed that such central channels should be regulated but without limiting the freedom of speech in accordance with extant constitution of each country. ITU-D is encouraged to come up with Recommendations on how social media can be harnessed during pandemic, epidemic or contagion since this is not a technical issue but more of developmental concerns.

A lot of countries have implemented partial and full lockdown depending on the phase they are in on COVID-19 pandemic. The importance of lockdown is to encourage people not to move around so that the COVID-19 can be contained and the spread mitigated. Offices asked their staff to work from home so that the businesses will not suffer and economic activities can continue. However good the intention is there is not standard around most of the work at home order (although some countries has started looking at this). This is one of the reasons why this paper is written to provide a way forward for the future for a better work at home during lockdown. There is need for rules and regulation guiding the work at home. The ICT facilities required as per minimum standards. The speed of internet should be provided for seamless connection and effective work at home. All these need to be standardized so that there is an acceptable standard of digital infrastructure that will enable work at home during lockdown.

The recent pandemic has changed the way we do a lot of things and one of such areas that changed is the way we seek for medical assistance. Telemedicine typically refers to the practice of medicine using technology to deliver care at a distance. A physician/clinician in one location uses a telecommunications infrastructure to deliver care to a patient at a distant site. The current pandemic has shown that telemedicine has come to stay as part of the society. Based on this, it is imperative that the ITU-T should start a new Work Item that will focus on the minimum infrastructure requirements, protocols, software requirements and hardware specifications that would be needed to have an effective and efficient system to support telemedicine. This will go a long way to assist both developing and underdeveloped countries on the requirement for telemedicine since this will help to bring services to patient in remote areas and other areas where the facilities can be centrally deployed for the need of the underserved and unserved areas. Patients can be monitored remotely using technology to gather patient data outside of traditional healthcare settings.

It is also important to have a standardized ITU-T Recommendation on the definition of e-readiness in which parameters to benchmark e-readiness of a country to combat pandemic or epidemic as the case may be, would be harmonized for a standardization purposes and such standardized matrices can be applied across board. A Question on this is hereby proposed to advance relevant work items on metrics, parameters and specifications and necessary signaling and protocols that are relevant to standardized e-readiness of member countries.

A lot of innovations and initiatives are coming up. There is need for government to create an enabling environment to support such innovative digital technologies all over the world. For example, healthcare workers need to be able to detect COVID-19 fast enough so as to protect them from being infected while mitigating further spread to others simultaneously. Thirteen projects of such will be delivered within the next 24 months according to the European Commission [15].

\section{Conclusion}

From the seven (7) Recommendations made above, it can be concluded that there is need to start benchmarking, lightly regulating (through the use of Guidelines and Frameworks) and standardizing the technologies used in combatting pandemic like COVID-19 with specific focus on the specifications and requirements that will give the minimal Quality of Experience as it is required for services to support the fight against pandemic or contagion which in most cases can be critical and the failure rate of such system is expected to be very low since such situation can be categorized as national emergency or global emergency as the case may be. The work of ITU in working on the identified Questions or Work Items will go a long way to assist countries in putting up an efficient Pandemic Management System with the aim of combatting the pandemic or epidemic.

Finally, the need for International Telecommunications Union, Standardizations Sector to start Question that will deal with Pandemic and epidemic has been successfully discussed and analyzed. It is expected that ITU-T will approve new Question in face of the upcoming World Telecommunications Standardization Assembly (WTSA), World Telecommunications Development Conference or through invitation of submission of new proposed Work Items to relevant Study Groups during Study Group Meetings.

\section{References}

[1] Koulopoulos, T. and Palmer, N. (2001) 'The X- economy: profiting from instant commerce', Texere, June. 
[2] Shapiro, C. and Varian, H. (1999) Information Rules: A Strategic Guide to the Network Economy, Harvard Business School Press, Boston, MA.

[3] World Health Organization, (2020) Sustainable Development Goals (SDGs) Source: https://www.who.int/sdg/targets/en/ [Accessed: 23rd June, 2020].

[4] United Nations Conference on Trade and Development (UNCTAD), (2019), Digital Economy Report 2019 Value Creation and Capture: Implications for Developing Countries, United Nations Publications, Geneva.

[5] Bethscheider, Gerhard (2015). Barney, Randall (Ed.). "Satellite is vital for a unified, global, E-Health system. An SES Techcom Services Perspective". World Teleport Association. Source: https://www.worldteleport.org/news/249029/Satellite-Is-VitalFor-A-Unified-Global-e-Health-System...-An-SES-TechcomServices-Perspective-.htm [Accessed: 12th April, 2020].

[6] O. J. Kunle, O. A. Olubunmi and S. Sani, Internet of things prospect in Nigeria: Challenges and solutions, (2017) IEEE 3rd International Conference on Electro- Technology for National Development (NIGERCON), Owerri, pp. 736-745.

[7] International Telecommunications Union, (2020) Telecoms, Coronavirus and keeping the networks running: Opinion Source: https://news.itu.int/telecoms- coronavirus-andkeeping-the-networks-running-time-for-leadership/ [Accessed: 12th April, 2020].

[8] Joaquin A. Blaya, Hamish S. F. Fraser, and Brian Holt, (2010), "EHealth Technologies Show Promise In Developing Countries", (2010) Health Affairs Vol. 29, No. 2: E-Health in the Developing World Available at: https://www.healthaffairs.org/doi/full/10.1377/hlthaff.2009.08 94 [Accessed: 1st July, 2020] 9 CNN (2020).
[9] https://edition.cnn.com/2020/04/04/asia/taiwan- coronavirusresponse-who-intl-hnk/index.html [Accessed: 15th April, 2020].

[10] C. Jason Wang, Chun Y. Ng and Robert H. Brook (2020), Response to COVID-19 in Taiwan Big Data Analytics, New Technology, and Proactive Testing Source: https://jamanetwork.com/journals/jama/fullarticle/27 62689? guestAccessKey=2a3c6994-9e10-4a0b-9f32cc2fb55b61a5\&utm_source=For_The_Media\&utm_medi $\mathrm{um}=$ referral\&utm_campaign $=\mathrm{ftm} \_$links\&utm_content $=\mathrm{tf}$ 1\&utm_term $=030 \overline{3} 20$ [Accessed: $\overline{3} 0$ th June, 2020 ].

[11] International Telecommunications Union (2020), COVID-19: How Korea is using innovative technology and AI to flatten the curve Source: https://news.itu.int/covid-19-how-korea-isusing- innovative-technology-and-ai-to-flatten-the-curve/ [Accessed: 7th April, 2020].

[12] Osong Health Technology Administration Complex (2019), Smart quarantine information system project, KCDC, Korea. Source: $\mathrm{http}: / /$ www.cdc.go.kr/contents.es?mid=a30301180000 [Accessed: 20th April, 2020].

[13] ITU (2020), COVID-19: How Korea is using innovative technology and $\mathrm{AI}$ to flatten the curve Source: https://news.itu.int/covid-19-how-korea-is-using-innovativetechnology-and-ai-to-flatten-the-curve/ [Accessed: 27th June, 2020].

[14] D. Phillips, (2005), “Texas 9-1-1: Emergency telecommunications and the genesis of surveillance infrastructure," Telecommunications Policy, vol. 29, pp.

[15] European Commission (2021): Digital health technologies addressing the pandemic. Source: https://ec.europa.eu/digitalsingle-market/en/digital-health-technologies-addressingpandemic [Accessed: 25th January, 2021]. 